Best practice and pitfalls in absolute structure determination

Linden, Anthony

DOI: https://doi.org/10.1016/j.tetasy.2017.07.010

Posted at the Zurich Open Repository and Archive, University of Zurich

ZORA URL: https://doi.org/10.5167/uzh-141601

Journal Article

Accepted Version

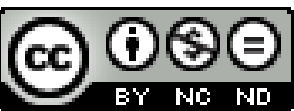

The following work is licensed under a Creative Commons: Attribution-NonCommercial-NoDerivatives 4.0 International (CC BY-NC-ND 4.0) License.

Originally published at:

Linden, Anthony (2017). Best practice and pitfalls in absolute structure determination. Tetrahedron: Asymmetry, 28(10):1314-1320.

DOI: https://doi.org/10.1016/j.tetasy.2017.07.010 


\title{
Best Practice and Pitfalls in Absolute Structure Determination
}

\author{
Anthony Linden \\ Department of Chemistry, University of Zurich, Winterthurerstrasse 190, CH-8057 Zurich, \\ Switzerland \\ anthony.linden@chem.uzh.ch
}

Dedicated to the memory of Howard D. Flack $(\dagger 2017)$

\section{Abstract}

In routine small-molecule single crystal structure determination, accurate absolute structure determination has sometimes been challenging. Developments in diffractometers, X-ray sources, detectors and software, along with new concepts for the elucidation of the absolute structure have seen the greatest advances in recent times. Nonetheless, determining the absolute structure of a crystal, particularly when only light atoms are present, requires some thought in the planning of the experiment in order to obtain the best possible data and some care in modelling the structure and interpreting the results so as not to draw incorrect or unsupported conclusions. Some practical recommendations for best practice and how to avoid pitfalls and misinterpretations are presented as a guide, particularly for those new to the field of crystal structure analysis. 


\section{Introduction}

$\mathrm{X}$-ray crystal structure determination is one of the few methods available for the reliable determination of the absolute configuration of molecules containing one or more stereogenic centres. Such knowledge can be important in many applications. Two examples are chemical synthesis, where the outcome and mechanism of a reaction must be elucidated to understand and predict future reactions involving that synthesis method, or in the pharmaceutical industry, where the benefits or dangers of a new chiral drug molecule may be dependent on its absolute or even relative configuration.

However, while determination of the gross crystal structure is normally quite straightforward these days, the extra information required to elucidate the absolute structure cannot always be obtained reliably, because the effect contributing this needed information, anomalous scattering, is small and its magnitude is dependent on the atomic number of the heaviest elements in the structure and the wavelength of the X-rays used. In the absence of anomalous scattering, the diffraction pattern is inherently centrosymmetric and the additional contribution of the anomalous scattering to the reflections intensities, which causes the needed differences in the intensities of Bijvoet pairs of reflections, is mostly small, compared with the intensities themselves. ${ }^{1,2}$ Over the years, different methods have been developed to utilise the anomalous scattering information as far as possible toward the determination of absolute structure, but the early success was often limited to structures containing heavy elements. The most significant advance in the utility and sensitivity of absolute structure determination came with the seminal work of Howard Flack in $1983,{ }^{3}$ in which a crystal structure is treated as an inversion twin and the relative twin fraction is refined. Some further fine tuning of this concept over the last 10 15 years has led to improvements in the precision of the absolute structure determination. ${ }^{4-6}$

In honour of the contributions of Howard Flack to this field (Fig. 1), this paper focuses on practical aspects of the determination of absolute structure during single-crystal X-ray structure determination, the interpretation of the results of such attempts, and some of the pitfalls of the technique for the unwary. As a co-editor and then main editor of Acta Crystallographica, Section $C$ for the last 20 years, one occasionally sees papers in which incorrect conclusions are based on misinterpretations of absolute structure determinations. Therefore, it is hoped that the discussion presented herein will be of benefit to those performing absolute structure determinations, or interpreting the reported results thereof. 


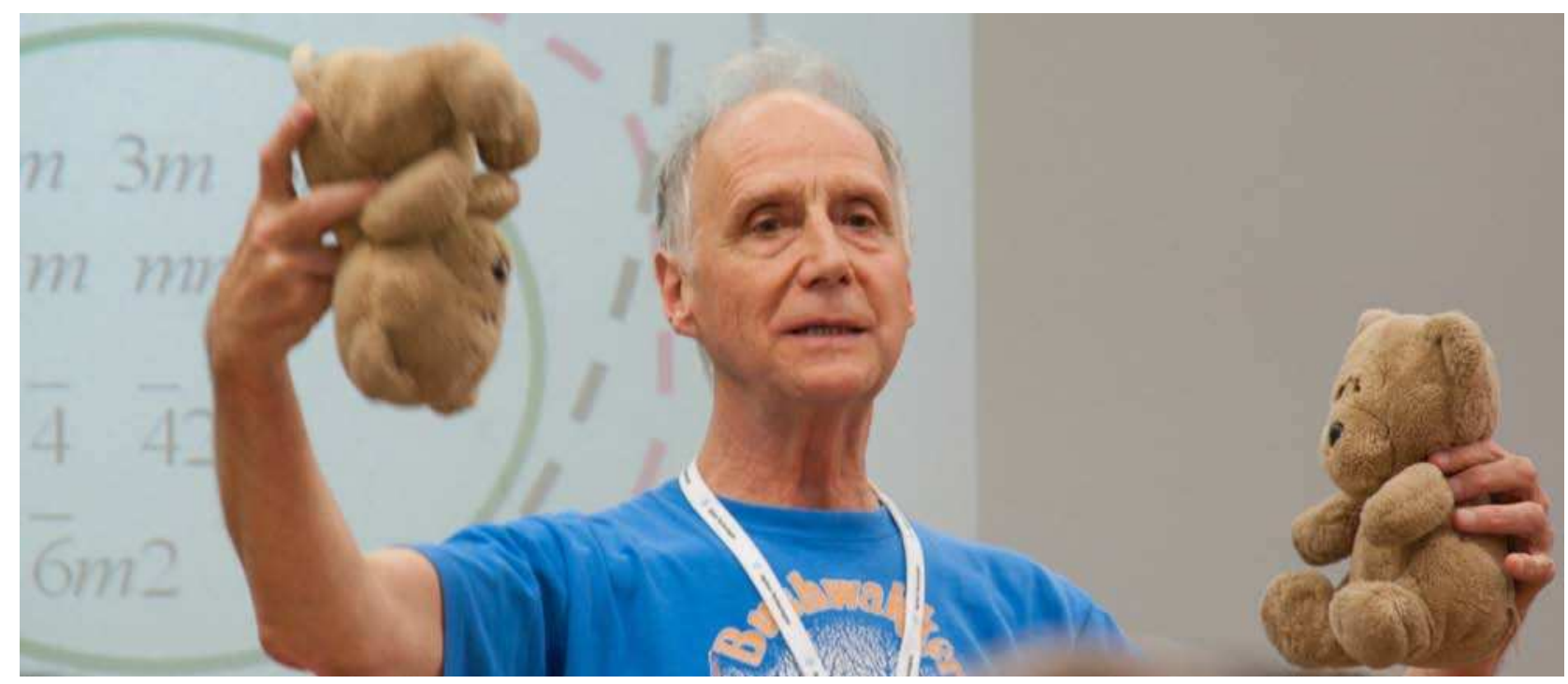

Figure 1. Howard Flack demonstrating symmetry using his signature teddy bears during a lecture on absolute structure at the Zurich School of Crystallography in June 2013. Photo: M. Wörle, ETH Zurich.

\section{Discussion}

\subsection{Some history}

A very good history of the various methods of absolute structure determination up to 2008 is given by Hooft et al. ${ }^{4}$ Up to then, the most important development was when, in 1983, Flack introduced the absolute structure parameter (colloquially now known as the Flack $x$ parameter) as a parameter that could be refined during least-squares model refinement in a crystal structure determination, where a value of zero suggested that the model represented the true absolute structure and a value of one suggested that the model needed to be inverted. ${ }^{3}$ This parameter had the advantage that an intermediate value might indicate inversion twinning. The Flack parameter, $x$, comes with a standard uncertainty (s.u.) of $u$, which can be derived during the refinement from the covariance matrix, and this s.u. is just as important in the interpretation of the success of the absolute structure determination as the Flack parameter itself. Obviously, a Flack parameter, $x(u)$, with its $x$ range of $0-1$ is meaningless if the value's standard uncertainty is on the same order of magnitude and one cannot determine the absolute structure convincingly in such a case. Flack and Bernardinelli subsequently wrote two key papers outlining in detail the statistical basis for the interpretation of the Flack parameter and its s.u. ${ }^{7,8}$ They give a quite conservative view, in that if the presence of inversion twinning cannot be excluded, an s.u. of 0.04 or less is required to be certain statistically that the model represents 
the true absolute structure. If it is known that a compound is enantiomerically pure, so that twinning is impossible (one needs to be certain that a tiny amount of the opposite enantiomorph in the bulk material is definitely not possible), then s.u.s up to around 0.1 may be sufficient. In practice, many people tolerate slightly larger s.u.s and still feel confident, but if you are likely to be fighting a case in court over that, it is better to err on the side of conservatism.

The magnitude of the s.u. of the Flack parameter is dependent on the strength of the anomalous scattering (resonant scattering) in the crystal and the X-ray wavelength used, so is smaller when heavy atoms are present and/or longer wavelengths are used. With Mo Ko radiation, it is usually difficult to obtain an s.u. small enough for the Flack parameter to be an unequivocal indicator of the true absolute structure when no element heavier than $\mathrm{Si}$ is present in the structure (the heavy element does not have to be in the molecule of interest itself). When $\mathrm{Cu}$ $K \alpha$ radiation is used, it is often, but not always, possible with good data to obtain a sufficiently small s.u. on the Flack parameter when the heaviest element is just oxygen. The recent advent of dual X-ray source diffractometers has made such experiments with $\mathrm{Cu} K \alpha$ radiation much more accessible routinely than before, because the effort associated with changing the X-ray source and the sensitivity optimisation of CCD detectors for Mo Ka X-rays, meant that many laboratories were unwilling to switch to $\mathrm{Cu}$ radiation when needed for absolute structure determination of light-atom structures. Nonetheless, even with $\mathrm{Cu} K \alpha$ radiation, the s.u. on the Flack parameter is sometimes still too large.

For about 20 years after the introduction of the Flack parameter, most people thought that absolute structure determination by use this method was about as good as we could do, but recent clever developments showed that even more information about absolute structure could be extracted out of the diffraction data when analysed carefully. The "Hooft parameter", $y(u),{ }^{4,9}$ based on Bayesian statistics, and Parson's $z$ parameter, $z(u),{ }^{5,6}$ based on a differences in a subset of Bijvoet reflections have helped to reduce the magnitude of the s.u. on the absolute structure parameter further. The reader is referred to the relevant papers for further details. Users of common structure refinement and analysis packages, such as, but not limited to, SHELXL,${ }^{10}$ CRYSTALS,${ }^{11,12}$ OLEX ${ }^{2},{ }^{13}$ and PLATON,${ }^{14,15}$ can easily obtain the Flack, Hooft and Parson's parameters. 


\subsection{Some definitions}

Three key terms are relevant in this discussion: absolute structure, absolute configuration and relative configuration. It is important to know which term is appropriate for any structural investigation at hand. If a molecule has one or more stereogenic centres, which can include axial chirality, and the sample is enantiomerically pure, one might wish to know the absolute configuration of the molecule, i.e. which enantiomorph one has and the correct "handedness" of all such stereocentres. If a molecule has two or more stereogenic centres, then one can talk about the relative configuration of the centres within the molecule. This has no bearing on whether the substance is enantiomerically pure or is a racemate. Molecules with different relative configurations are stereoisomers. In a crystal structure determination, if the space group is non-centrosymmetric, whether the space group be chiral or not (see below), it is possible to determine the absolute structure of the contents of the crystal. It is not necessary that the structure contains chiral molecules in such a case, as the packing of achiral molecules can still result in a chiral structure. However, if the non-centrosymmetric space group is, in addition, chiral and the molecule contains one or more stereogenic centres, then the substance is enantiomerically pure (except in the case of inversion twinning, see §3.4.7) and as a consequence of the determination of the absolute structure, the absolute configuration of the molecule is also established. If the space group is centrosymmetric or non-centrosymmetric with symmetry operations of the second kind (mirrors, glide planes, rotary inversion centres; see §3.4.2), then relative configuration is the sole aspect one can discuss because if stereogenic centres are present, the crystal must have a racemic mixture of the substance. A more detailed treatise on these concepts is given by Flack and Bernardinelli. ${ }^{7}$

\subsection{Good practice for obtaining the best results}

\subsubsection{Data collection strategy}

The advice for anyone undertaking any crystal structure analysis is that one should try to obtain the best data reasonably possible for the purpose at hand right from the outset. That might seem to go without saying, but it is often very tempting with a crystalline sample of borderline quality obtained from the first crystallization attempt to try to push on in the hope of a quick result, rather than trying for better crystals right away. Many problems and wasted hours struggling with a not-so-good structure refinement might be avoided if good data are 
collected at the start. This means trying to find the best crystal possible and not be satisfied with second best. If the first crystallization attempt produces mediocre crystals, ask yourself honestly: is this the best I can do, or might another crystallization attempt help? Sure, there are some compounds that never seem to crystallize well, but it is unwise to go with the first batch if the crystals look poor, when you do not yet know whether or not a little more effort at this stage might produce a superior sample.

Collecting sufficient data is also important. On modern diffractometers, it is easy to collect data with high redundancy (large excess of reflections over the symmetry unique set, such as a 5:1 or higher ratio) without requiring a huge amount of additional time. The advantages of redundant data are that the mean intensity of a set of equivalents is likely to be closer to the true intensity, and the "multi-scan" method of applying absorption corrections and frame scaling works best with a high redundancy of reflections and their symmetry-equivalents. It is unfortunate that some practitioners are forced to confine their data collection strategy to a fixed block of time by the practice of their facility. One never knows beforehand if a particular sample will be strongly or weakly diffracting and it is better to spend the time required to collect highly redundant data with good counting statistics, no matter how long the data collection needs, than to squeeze the data collection into a fixed number of hours by sacrificing redundancy and/or intensity statistics.

In the case of absolute configuration determination, the contribution of anomalous scattering to the reflection intensities is usually small, so for the best chance of success in obtaining an accurate and precise determination, particularly when only small or modest anomalous scattering is present, it is vital to have the best quality data possible in a sufficient quantity. Any "noise" introduced into the intensities because of crystal quality issues can be detrimental to the success of the experiment mainly because of the decrease in precision of the absolute structure parameter (but see the comments in §3.4.8). In addition, one should try to collect a as close to a full set of Friedel or Bijvoet pairs of reflections as possible. It should be noted that some data collection software, such as CrysAlisPro, ${ }^{16}$ assumes a centrosymmetric structure when calculating the data collection strategy, so the "non-centrosymmetric flag" should be activated to ensure that the data collection includes as many Friedel or Bijvoet pairs of reflections as possible.

\subsubsection{Data reduction}


The data reduction step is equally as important as having a good crystal, especially when the material is strongly absorbing. While the default "absorption correction" and frame scaling carried out by modern diffractometer software is the empirical "multi-scan" type based on spherical harmonics, ${ }^{17}$ which works very well in most cases, even with strongly absorbing materials, provided the data set has sufficiently high redundancy, a further step of applying a numerical or analytical absorption correction, which requires indexing the crystal faces and measuring its dimensions, can yield improvements in data quality when absorption is strong. It is recommended that one spends at least a little time trying the various correction options in test repeats of the final data reduction steps in order to find the optimal correction and scaling conditions. The performance of each of these tests can be compared initially in terms of $R_{\text {int }}$ for merging symmetry-equivalent reflections and the magnitudes of the residual electron density peaks in the final refinement.

\subsubsection{How to improve the precision of the absolute structure parameter}

If the anomalous scattering power of a compound is weak and the s.u. on the absolute structure parameter is therefore large, what can be done to improve the precision of the absolute structure parameter so that a definitive conclusion can be drawn? There are several possibilities to consider, but the feasibility of any of them depends on the availability of the substance and the possible chemical transformations that might be made. These suggestions are aimed more at enabling the determination of the absolute configuration of a chiral molecule, than the absolute structure in an achiral, but polar non-centrosymmetric space group.

(a) If only $\mathrm{Ag} K \alpha$, Mo $K \alpha$ or $\mathrm{Ga} K \alpha$ radiation has been used so far, try $\mathrm{Cu} K \alpha$ radiation. $\mathrm{Cu}$ radiation gives the strongest anomalous scattering signal when the heaviest element in a compound is just oxygen. However, even $\mathrm{Cu}$ radiation does not always give a precise enough value for the absolute structure parameter, especially when the oxygen content is low or the compound is a pure hydrocarbon, or when the crystal quality is low.

(b) Co-crystallise with a species of known absolute configuration. This reduces the problem to one of relative configuration and one does not even have to worry about the precision of the absolute structure parameter at all. Of course, mixtures of compounds do not always form co-crystals, so some patience and trials are needed.

(c) Co-crystallise with a species containing a heavy atom. It is not necessary that the strong anomalous scatterer be part of the molecule of interest, it only needs to be in the crystal structure. If one is lucky, serendipitously obtaining co-crystal containing a solvent molecule like $\mathrm{CH}_{2} \mathrm{Cl}_{2}$ can solve the problem. 
(d) Make a derivative of the compound that incorporates a species of one of the types mentioned in (b) and (c). This requires that you have sufficient compound to play with, that you do not mind losing the original compound because of the transformation, if it is irreversible, and that a suitable derivatization reaction is feasible.

(e) If the compound has acidic or basic sites, maybe it could be protonated or deprotonated with a base or acid containing a suitable heavy counter-ion, so that a salt with sufficient anomalous scattering power is formed. The caveats of (d) apply here, too.

\subsection{Mistakes and misinterpretations}

\subsubsection{Ignoring the standard uncertainty of the absolute structure parameter}

It is folly to conclude from a refinement where the final model produces an absolute structure parameter near zero and the inverse model gives a value near one, that the absolute structure has been determined unequivocally, while ignoring that fact that the s.u. on the value is too large. One cannot simply ignore the magnitude of $u$. At the $3 \sigma$ confidence limits usually used in statistical analysis, a Flack parameter of $0.0(3)$ cannot be distinguished statistically from 1.0 because the $x$ parameter could have a value of $0.0+(3 \times 0.3)=0.9$, thus indicating the opposite absolute structure. As indicated by Flack \& Bernardinelli, ${ }^{8}$ and mentioned here in $\S 3.1$, if it is known from the origin of the compound that there is no possibility of inversion twining, a Flack parameter like 0.0(1) or 0.02(9) would be perfectly convincing, but if there is a chance of inversion twinning, one needs a smaller s.u. in order to exclude the possibility of a racemic twin, so a value like $0.02(4)$ would be suitable. An s.u. of 0.04 is considered conservatively by Flack \& Bernardinelli to be the upper limit in this latter case.

The Parson's $z(u)$ parameter is interpreted in exactly the same way. ${ }^{6}$ The Hooft parameter, ${ }^{4}$ $y(u)$, can be calculated and compared with $x(u)$ and $z(u)$ directly in PLATON. ${ }^{14}$ However, $y(u)$ is only calculated for convenience of this comparison. The real information coming from the Bijvoet Pair analysis in PLATON is the probabilities, $P$, that the true absolute structure has been found or that the opposite absolute structure or inversion twinning is possible. ${ }^{4}$ Values given are $P 2$ (true), $P 3$ (true), $P 3$ (rac-twin) and $P 3$ (false), which are, respectively, the probabilities that the model represents the true absolute structure if inversion twinning is not possible, if twining is possible, that the structure is an inversion twin (referred to as a racemic twin in the program), and the probability that the model has the wrong absolute structure. In 
practice, if $P 2$ or $P 3$ deviate from 1.000 even in the third decimal place, one might need to be cautious.

\subsubsection{Ignoring the space group symmetry - absolute configuration versus absolute structure}

An enantiomerically pure compound can only crystallize in one of 65 chiral (Sohncke) space groups. These are space groups that only have translations and rotations, plus their combination, screws, as symmetry operations. All of the other 165 space groups involve symmetry operations of the second kind, i.e. a mirror or inversion operation (with their derivatives, the glide planes and rotary inversion axes), which necessarily means that crystals in these space groups composed of molecules with one or more stereogenic centres are racemic.

Some misinterpretations occasionally arise because of a lack of understanding or consideration of space group symmetry and what one sees in a crystal structure analysis. One incorrect conclusion sometimes drawn is that the molecule shown in a molecular structure diagram, which represents perhaps the contents of the asymmetric unit or less, must be representative of the stereochemistry and absolute configuration of all molecules in the crystal, because the person does not realise the implications of space group symmetry. A crystal conforming to a centrosymmetric space group must consist of a racemic mixture of enantiomorphs, because of the presence of the inversion symmetry operation $-x,-y,-z$. Others might think that just having a non-centrosymmetric space group is sufficient for being able to establish the absolute configuration of a molecule with stereogenic centres, but, once again, some noncentrosymmetric space groups still include mirror, glide or certain rotary inversion symmetry. As a general guide, space groups which can accommodate an enantiomerically pure chiral molecule are only those that do not have a lowercase letter, $m, a, b, c, d, e, n$, or $\overline{1}, \overline{3}, \overline{4}$, or $\overline{6}$ in the space group symbol. Non-centrosymmetric space groups with any of these symbols will necessarily have a racemic mixture of the compound if a chiral molecule is present, despite the absence of a centre of inversion (space groups with the symbols $\overline{1}$ or $\overline{3}$ are always centrosymmetric).

On the other hand, non-centrosymmetric crystal structures that impose the necessity of a racemic mixture of chiral molecules, when present, or even those that contain achiral molecules can still have a specific absolute structure, because there is still a polar axis (i.e. a 
distinct "top" and "bottom" of the physical crystal) and the correct determination of the direction of that axis, i.e. the absolute structure, can be achieved or may even be necessary in order to avoid bias of the atomic coordinates in the refined model (see §3.4.9). Even achiral molecules, such as $\mathrm{SiO}_{4}$, can crystallise in a chiral space group and, again, the determination of the true absolute structure, as distinct from the absolute configuration, is still advisable.

A related problem is the refinement of a crystal structure in a non-centrosymmetric space group when the corresponding higher-symmetry centrosymmetric space group is the correct one. This might occur because the practitioner believed incorrectly that the compound had to be enantiomerically pure, so chose the chiral space group corresponding as closely as possible with the observed systematic absences was chosen, or the structure solution for some reason did not "pop out" easily with the centrosymmetric space group, but was found quickly in a non-centrosymmetric space group and the possibility of missed symmetry was not checked for later on. In such a case, the absolute structure factor should come out to 0.5 , because the compound would be racemic. A further issue with refining a model in a space group with lower symmetry than the true space group can be that the atomic positions might be highly biased because of correlations in the least-squares refinement procedure, which can lead to incorrect conclusions about bond lengths and other geometric parameters. ${ }^{17}$

\subsubsection{Misunderstood chemistry}

While not directly an issue of absolute structure determination per se, a related misunderstanding can arise when the chemist claims to have delivered an enantiomerically pure chiral compound for analysis, and has the optical rotation results to support the claim, but the crystal structure shows that the compound in the chosen crystal is definitely racemic. In such a case, the chemist may be tempted to assume, or even insist, that the model must represent the absolute configuration of a single enantiomorph, as mentioned in §3.4.1. This has been encountered this in my own laboratory several times. Only after careful discussion with the chemist is it revealed that the reaction or chemical work-up actually did not deliver a $100 \%$ enantiomerically pure compound, but that there was a small amount, say $5 \%$, of the opposite enantiomer present. What has happened is that during crystallization, the $5 \%$ has crystallized as a racemic mixture to give just a few nice crystals, which is generally favoured because centrosymmetric structures tend to crystallize better than non-centrosymmetric ones (anecdotally), while the bulk of the enantiomerically pure compound has crystallized poorly or even as a sludge, so the crystallographer picks out the nice-looking crystal and the result is a 
centrosymmetric crystal structure. The moral of this story is that one must keep in mind that the composition of the single crystal used for the structure determination is not necessarily representative of the composition of the bulk material and that purity of the bulk should always be confirmed by other experiments or spectra. A powder X-ray diffraction pattern cannot distinguish enantiomorphs, of course, although it might reveal mixtures of enantiopure and racemic crystals (and other impurities), because the two types of crystals are likely to have different unit cell parameters.

\subsubsection{Illegal unit cell transformations}

It is quite common that the unit cell parameters initially found need to be transformed in order to conform with the standard setting for the space group concerned. These days, this is often taken care of automatically during the data reduction or structure solution stages, so that the user is unaware of the actual transformation operation being applied. However, situations can occur where the user needs to do the unit cell transformation manually. Most often, this involves interchanging two of the unit cell axes, or a cyclic rotation of all three. For example, converting $P 2_{1} / a$ to $P 2_{1} / c$ requires the unit cell $a$ and $c$ axes to be swapped. In doing so, it is essential that the unit cell is not converted from a standard "right hand" one (so defined if the unit cell is viewed such that the unit cell $a$-and $b$-axes are in the plane of the page with the $a$ to- $b$ rotation through $\gamma^{\circ}$ anticlockwise and the $c$-axis coming out of the page towards the viewer) to a "left hand" one. This is not only inappropriate in all transformations, but is critical for an absolute structure determination, because the illegal transformation would also invert the structure and lead to a misinterpretation of the absolute structure and absolute configuration of a molecule. A transformation involving swapping two of the unit cell axis must have one or three, but not two, negative signs in the transformation matrix, e.g. the $P 2_{1} / a$

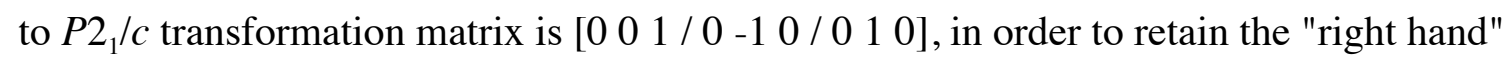
arrangement, while a cyclic rotation of all three axes should have none or two negative signs, e.g. [0 $01 / 2100 / 010]$ for an $a b c$ to $c a b$ interchange. Modern software often warns the user when an attempt is made to create a "left-handed" unit cell, but this might not always be the case, so the user needs to remain aware of this trick. The problem is particularly prevalent when transformations of orthorhombic unit cells are carried out by the unwary.

\subsubsection{Merging data in the incorrect point group}


It is common practice today not to merge the equivalent and duplicate reflections in a data set prior to use in the structure refinement, because the structure refinement software usually takes care of this. This is a good thing. One advantage of this practice is that the original unmerged data are then still available in the archived CIF if any interested person wishes to try a refinement in a different space group or crystal system, without any assumption of the lattice symmetry having been imposed on the data beforehand. If it is desired to pre-merge the data for some reason and the structure is non-centrosymmetric, it is important that the correct point group is used for determining the symmetry-equivalent reflections during the merging, otherwise the effect of the anomalous scattering can be negated and no or erroneous conclusions might be drawn about the absolute structure. For example, merging the data for any non-centrosymmetric structure in any centrosymmetric point group, like $\overline{1}, 2 / \mathrm{m}, \mathrm{mmm}$, 4/m, 4/mmm, $m-3 m$, etc., averages the Bijvoet pairs, thus scrambling all potential information about the absolute structure and the Flack parameter would become 0.5 regardless of the space group. In non-centrosymmetric monoclinic space groups, the chiral space groups have point group 2 in which the intensities of the $h k l$ and $\bar{h} k \bar{l}$ reflections are strictly equivalent, while $h-k l$ is the mate of the Friedel opposite $\overline{h k} \bar{l}$ and so is not equivalent in intensity to the former two. Merging $h k l$ and $h \bar{k} l$ in such a case is equivalent to applying the symmetry of point group $m$ and, again, this would scramble the potential information about the absolute configuration of a chiral molecule. In orthorhombic space groups, one must be careful about the distinction between equivalent reflections in point group 222 (chiral) and $\mathrm{mm} 2$ (non-centrosymmetric, but achiral; see §3.4.2). Some years ago, a version of a diffractometer control software was merging data in the incorrect point groups and unwittingly destroying any information about the absolute structure. Presumably, no instrument is still running that version today, but if one consistently obtains Flack parameters near 0.5 for many supposedly enantiomerically pure compounds, be wary.

\subsubsection{Inverting the model - sometimes space group changes are needed}

If the absolute structure parameter suggests a structure model should be inverted, this can usually be done simply by inverting the coordinates of the atoms in the refinement model. The operation $-x,-y,-z$ can be applied, or something like $1-x, 1-y, 1-z$ might be better so that the atomic coordinates remain within the base unit cell. However, there are 11 pairs of space enantiomorphic groups (related by inversion) where simply inverting the atomic coordinates is not sufficient; the space group must also be changed to the other member of the pair. The 
consequence of forgetting this is that the refinement does not work sensibly at all any more, so failure to obtain a sensible structure refinement is usually the result for the unwary, rather than there being a risk of deducing the wrong absolute structure. The pairs of space groups are those containing screw axes where more than two operations of the screw are necessary in order to return to the original position, but in the next unit cell along: namely $P 4_{1}$ and $P 4_{3}$, $P 4_{1} 22$ and $P 4_{3} 22, P 4_{1} 212$ and $P 4_{3} 212, P 3_{1}$ and $P 3_{2}, P 3_{1} 21$ and $P 3_{2} 21, P 3_{1} 12$ and $P 3_{2} 12, P 6_{2}$ and $P 6_{4}, P 6_{1}$ and $P 6_{5}, P 6_{2} 22$ and $P 6_{4} 22, P 6_{1} 22$ and $P 6_{5} 22$, and $P 4_{1} 32$ and $P 4_{3} 32 .^{19}$

\subsubsection{Correct handling of inversion twinning}

The most commonly used refinement program, SHELXL, ${ }^{10}$ in the versions from 2013 onward, calculates the absolute structure parameter post-refinement. The refinement is done under the assumption that the model represents $100 \%$ of the true absolute structure and the output value of the absolute structure parameter will either be the Flack parameter itself, $x(u)$, or the Parson's parameter based on quotients, $z(u)$, whichever has the most precise value (smallest standard uncertainty, $u$ ). This is fine when $x$ or $z$ are within $3 u$ of zero, which is the case when the structure clearly has a single handedness (enantiomorphically pure for chiral compounds) or polar axis direction, or the anomalous scattering power is so weak that $u$ is too large for any definitive conclusions to be drawn about the true absolute structure, in which case the choice of absolute structure is arbitrary or can be aligned with the known chirality of the compound where that is known unequivocally from the chemical synthesis route (see §3.4.1). However, what if $x$ or $z$ deviate by more than $3 u$ from zero and $u$ is small, such as a value of $0.41(3)$. This is usually indicative of some degree of inversion twinning. Refining in the default way ignores the twinning in the refinement, so the least-squares refinement has not reached the true minimum and the consequence can be that the $R$-factor is slightly too high and, more importantly, the positions of the heaviest atoms can be biased slightly away from their true positions. ${ }^{20}$ It is important in such cases to refine the model properly as an inversion twin. In SHELXL, this means using the TWIN and BASF instructions. Partial inversion twinning with unequal domains is possible, so an inversion twin does not necessarily have to yield a Flack parameter of exactly 0.5 . A corollary to this is that an incorrect structure model can influence the absolute structure factor. In a chlorobisphosphinegold(I) complex, the incorrect position for the Cl-atom led to a Flack parameter of 0.21(2), an $R$-factor of 0.04, and a residual electron density maximum of $1.06 \mathrm{e} \AA^{-3}$, which seems to be a reasonable result and might lead one to conclude that an inversion twin was present. For the correct model, where the $\mathrm{Cl}$-atom was 
moved to the opposite side of the Au-atom where the residual peak was, the Flack parameter was $0.01(1)$ with an R-factor of $0.025 .^{20,21}$

It is important to note that it is possible to give an incorrect instruction combination to SHELXL for twin handling and the unwary might thereby draw incorrect conclusions. If the TWIN instruction is used, the BASF instruction must also be present. If BASF is missing, the program runs nonetheless, but the absolute structure parameter is not refined and instead is assigned some default value. For SHELXL-2014 and later, this is 0.5 (equal twin fractions are assumed). For earlier versions of SHELXL, the value was assigned as 0.0 and thus might have misled some users into thinking they had determined the correct absolute structure! In both cases, no s.u. is given. If you encounter a Flack parameter without an s.u. in a structure report or data deposited in a database (e.g. a CIF), be very sceptical.

\subsubsection{The effect of random errors in the reflection intensities}

One might imagine that because the anomalous scattering contribution to the intensities is small, significant errors in intensity measurements might scramble the absolute structure information, leading at least to a large value of the s.u on the absolute structure parameter, which might then make it impossible to draw definitive conclusions about the absolute structure. In other words, poor crystals and poor data reduce the chances of a reliable determination. To some extent, this is correct, as one learns from experience, but a recent series of tests showed that the absolute structure parameter and its s.u. is very robust, particularly when strong anomalous scatterers are present. ${ }^{22}$ Random errors were added to diffraction data sets by adding to or subtracting from each intensity a random percentage of its intensity to give a variation of up to $I \pm n$, where $n$ was a set maximum percentage for each trial. Generally, for structures containing reasonably strong anomalous scatterers, such as $\mathrm{Cl}, \mathrm{P}$ and/or $\mathrm{Au}$ (Mo $K \alpha$ radiation), $n$ could be up to $30-40 \%$ or even higher before a significant increase in the s.u. of the absolute structure parameter occurred, while the parameter itself did not deviate markedly from zero. Thus, significant random errors in the intensities seem to be able to be tolerated without unduly affecting the absolute structure parameter or its precision. On the other hand, the affect of systematic errors was not investigated.

\subsubsection{What if the incorrect absolute structure is refined?}


If the sample does not contain molecules with stereogenic centres, but nonetheless has crystallized in a chiral or even an achiral non-centrosymmetric space group, or a racemic mixture has crystallized in an achiral non-centrosymmetric space group, maybe there is little interest in the absolute structure. Can the absolute structure evaluation then be ignored, even if the absolute structure parameter clearly suggests that the structure model ought to be inverted? The same arguments as those given above for inversion twinning in $\$ 3.4 .7$ apply. In particular, in the presence of significant anomalous scatterers in the structure, the potential bias in atomic positions and thus the geometric parameters, which might result from the refinement not being properly minimized, should not be ignored.

\subsubsection{What happens if SQUEEZE is used?}

In recent years, the use of $S Q U E E Z E^{15}$ in PLATON, or the solvent mask function of $O L E X 2^{13}$, to avoid having to model disordered solvent or uninteresting disordered counter-ions in a structure has grown in popularity. In any situation where it is important to determine unequivocally the absolute configuration of a compound or the absolute structure, it is best to avoided using SQUEEZE altogether and to attempt to model the disordered solvent as well as possible, even if it cannot be done perfectly. At least one can then be sure that the contributions to the intensities of the anomalous scattering atoms have not been modified in an unexpected way by SQUEEZE. Importantly, what if the sole source of the anomalous signal lies within the disordered solvent region, such as would occur in the chloroform solvate of an organic molecule with just $\mathrm{C}, \mathrm{H}, \mathrm{N}, \mathrm{O}$ atoms? SQUEEZE-ing out the solvent molecule may have unexpected consequences for the absolute structure parameter because the contributions to the anomalous scattering have mostly been removed. A recent paper addresses this issue with an interesting supplemental procedure, which might be used in conjunction with SQUEEZE. ${ }^{23}$

\subsubsection{Twinning in general}

If a structure is twinned through operations other than inversion twinning, be it merohedral or non-merohedral twinning, some care should be take in defining the absolute structures of the twin domains by choosing the twin matrices appropriately. This is particularly important for an enantiomerically pure compound, where inversion of one domain during data reduction might be needed in order to give both major domains the correct absolute structure. ${ }^{24-26}$ 


\section{Conclusions}

Developments in the methodology of absolute structure determination are ongoing and absolute structure determination is a reliable and amenable nowadays to even light atom structures, provided one is careful with the planning of the experiment and interpretation of the results obtained. In recent years, Flack \& co-workers extended their work to the investigation of plots of the averages versus the differences of the intensities of Friedel opposites as a means of distinguishing centrosymmetric from non-centrosymmetric crystal structures and for validation of an absolute structure determination, particularly when materials that have weak anomalous scattering are at hand. ${ }^{27-29}$

In this treatise, an attempt has been made to outline the current state of the art in absolute structure determination and to give some guidelines about best practice for obtaining the most reliable absolute structure or absolute configuration determination possible for a given sample. Some of the pitfalls and misinterpretations that occasionally appear in the literature have also been highlighted in the hope that readers, especially those new to crystal structure analysis, may avoid these issues in the future. Related articles by Thompson and Watkin, and Flack are well worth reading. ${ }^{29,30}$

The most important message is to be cautious about the interpretation of absolute structure parameters when their standard uncertainties are on the high side. While one might be excited and eager to get a firm confirmation about the absolute configuration of a new molecule, conservatism is urged when the precision of the result makes the confidence in its interpretations borderline. It can be disastrous, or at best a sad waste of time, to "get it wrong" and plough on with a false assumption because one pushed the boundary too far. This applies not just for the crystal structure determination itself, but also for the chemistry or other scientific investigation that builds on the result.

\section{Acknowledgements}

Conflicts of interest: none 
This work did not receive any specific grant from funding agencies in the public, commercial, or not-for-profit sectors.

\section{References}

1. Guinier, A. X-ray Diffraction in Crystals, Imperfect Crystals, and Amorphous Bodies; Dover: New York, 1994.

2. Glusker J. P.; Lewis, M; Rossi, M. Crystal Structure Analysis for Chemists and Biologists; VCH Publishers: New York, 1994.

3. Flack, H. D. Acta Crystallogr. 1983, A39, 876-881.

4. Hooft, R. W. W.; Straver, L. H.; Spek, A. L. J. Appl. Crystallogr, 2008, 41, 96-103.

5. Parsons, S.; Flack, H. D. Acta Crystallogr. 2004, A60, s61.

6. Parsons, S.; Flack, H. D.; Wagner, T. Acta Crystallogr. 2013, B69, 249-2591.

7. Flack, H. D.; Bernardinelli, G. Acta Crystallogr. 1999, A55, 908-915.

8. Flack, H. D.; Bernardinelli, G. J. Appl. Crystallogr. 2000, 33, 1143-1148.

9. Hooft, R. W. W.; Straver, L. H.; Spek, A. L. J. Appl. Crystallogr, 2010, 43, 665-668.

10. Sheldrick, G. M. Acta Crystallogr. 2015, C71, 3-8.

11. Betteridge, P. W.; Carruthers, J. R.; Cooper, R. I.; Prout. K.; Watkin, D. J. J. Appl. Crystallogr. 2003, 36, 1487.

12. Cooper, R. I.; Watkin, D. J. Flack, H. D. Acta Crystallogr. 2016, C72, 262-267.

13. Dolomanov, O. V.; Bourhis, L. J.; Gildea, R. J.; Howard, J. A. K.; Puschmann, H. J. Appl. Crystallogr. 2009, 42, 339-341.

14. Spek, A. L. Acta Crystallogr. 2009, D65, 148-155.

15. Spek, A. L. Acta Crystallogr. 2015, C71, 9-18.

16. CrysAlisPro, Rigaku Oxford Diffraction, Abingdon, UK, 2017, https://www.rigaku.com/en/products/smc/chrysalis

17. Blessing, R. H. Acta Crystallogr. 1995, A51, 33-38.

18. Flack, H. D.; Bernardinelli, G.; Clemente, D. A.; Linden, A.; Spek, A. L. Acta Crystallogr. 2006, B62, 695-701.

19. Looijenga-Vos, A.; Buerger, M. J. Space-Group Determination and Diffraction Symbols; Hahn, T, Ed. International Tables for Crystallography, Vol. A. Springer: The Netherlands, 2005; pp. 44-54.

20. Flack, H. D. Private communication, 2015. 
21. Larsonneur, A.-M.; Turpin, R.; Castan;, P.; Bernardinelli, R. Inorg. Chim. Acta 1994, 227, 85-90.

22. Linden, A.; Müller, M. J. Unpublished results, University of Zurich, 2015.

23. Cooper, R. I.; Flack, H. D.; Watkin, D. J. Acta Crystallogr. 2017, C73, submitted.

24. Herbst-Irmer, R.; Sheldrick, G. M. Acta Crystallogr. 1998, B54, 443-449.

25. Herbst-Irmer, R.; Sheldrick, G. M. Acta Crystallogr. 2002, B58, 477-481.

26. Guzei, I.; Herbst-Irmer, R.; Munyaneza, A.; Darkwa, J. Acta Crystallogr. 2012, B68, $150-157$.

27. Flack, H. D.; Sadki, M.; Thompson, A. L.; Watkin D. J. Acta Crystallogr. 2011, A67, 2134.

28. Parsons, S.; Pattison, P.; Flack, H. D. Acta Crystallogr. 2012, A68, 736-749.

29. Flack, H. D. Acta Crystallogr. 2013, C69, 803-807.

30. Thompson, A. L.; Watkin D. J. Tetrahedron: Asymmetry, 2009, 20, 712-717. 